\title{
Evoked potentials and stages of information processing: Methods of analysis
}

\author{
JONATHAN VAUGHAN, DOUGLAS J. HERRMANN, and GREGORY BELL \\ Hamilton College, Clinton, New York
}

\begin{abstract}
The use of principal components analysis (PCA) for the study of evoked-response data may be complicated by variations from one trial to another in the latency of underlying brain events. Such variation can come from either random intra- and intersubject variability or from the effects of independent variables that are manipulated between conditions. The effect of such variability is investigated by simulation of these latency-varying events and by analysis of evoked responses in a behavioral task, the Sternberg memory search task, which is well known to generate variation in the latency of brain events. The results of PCA of within-subjects differences in these two situations are plausibly related to underlying stages of information processing, and the technique may augment reaction time data by providing information on the time of occurrence as well as the duration of stages of information processing.
\end{abstract}

The event-related potential has been used by a number of investigators in recent years to supplement reactiontime measurements during performance on cognitive tasks (Coles, Donchin, \& Porges, 1986; Donchin, 1984). In one approach, evoked responses are related to cognitive stages of information processing by measurement of the latency of peaks in the averaged evoked response, often together with reaction-time measurements (e.g., Kutas, McCarthy, \& Donchin, 1977; McCarthy \& Donchin, 1981; Renault, Ragot, Lesevre, \& Remond, 1982). Such observations demonstrate that different stages of processing are affected by different variables. For example, P300 (a positive deflection of the averaged evoked potential with latency of $300 \mathrm{msec}$ or longer) has been associated with several aspects of active cognitive processing of stimulus information, such as stimulus evaluation (Pritchard, 1981). Peak measurements presuppose, of course, that the brain activity that is associated with each cognitive stage (hereafter referred to as an event) is accompanied by observable peaks in the evoked-response waveform, and that these peaks are best characterized by the latency or amplitude of the locally maximum excursion of the recorded voltage from baseline.

In another approach to characterizing evoked responses, principal components analysis (PCA) has been applied to evoked responses obtained during tasks of the additive factors design (Chapman, McCrary, \& Chapman, 1981;

The simulation data reported here were generated with the assistance of Mark Yemen. The behavioral data were acquired as part of Gregory Bell's senior honors thesis at Hamilton College. The research was supported in part by the Hamilton College Faculty Research Fund. Portions of the behavioral data reported here were presented at the meetings of the Society for Psychophysiological Research in October 1986 and of the Psychonomic Society in November 1986. The precise details of the analyses performed here differ from those presented earlier in minor ways that do not affect the conclusions to be drawn.

Send reprint requests to Jonathan Vaughan, Department of Psychology, Hamilton College, Clinton, NY 13323.
Donchin \& Heffley, 1978). PCA requires no a priori identification of particular peaks, and comparisons across conditions are not limited to a few arbitrary characteristics of the waveform. Rather, the technique summarizes the data from each of a large number of individual observations as a linear combination of a few components, each of which represents a pattern of correlated differences across observations. In the most commonly used method of applying PCA, the average waveforms for several within-subjects conditions are combined to provide the corpus of raw data that is submitted to PCA. Typically, a few components describe a large proportion of the observation-to-observation variability, and the scores of some of these components vary in a consistent (i.e, statistically significant) manner from one experimental condition to another. Although task variations implicitly affect cognitive processes and brain events and empirically affect the components extracted by PCA from evoked responses, it cannot be claimed that the event and component are necessarily identical. Logically, the events associated with a particular process might affect none, one, or several of the components extracted by PCA of within-subjects differences in the evoked response. In particular, variations in the scores for a component cannot be taken in every case as the variation in the amplitude of an underlying brain event, stationary in time, that varies in amplitude from one condition to another, although it may be possible to do so in particular circumstances (e.g., Chapman et al., 1981).

An often unstated assumption in evoked-response research is that the sequence of brain events in successive trials is similar in latency on every trial that contributes to the average (referred to as the stationarity assumption). The assumption is, of course, central to the logic of the averaging process: signal, in the evoked-response waveform in each trial, is time locked with the stimulus that initiates each sampling period and is additive across the 
averaged trials, whereas noise is not time locked and thus is uncorrelated across trials.

The purpose of the present paper is to explore the consequences of violations of the stationarity assumption. We can evaluate the robustness of the assumption for situations of particular interest to psychologists by briefly examining the sources of variation in the most commonly used dependent variable, reaction time. Then, having demonstrated that the stationarity assumption is not strictly met in many experimental situations and is frankly violated in others, we consider the consequences of its violation by using principal components analysis to examine both simulated data and behavioral data (from the Sternberg memory search task, 1966, 1969) that violate it.

There are two sources of latency variation in reaction time in tasks that require speeded overt responses. First, reaction time can be observed to vary from one trial to another (random latency variation). In addition to this random variation, reaction time varies in response to independent variable manipulation (experimental latency variation). Within the information-processing tradition, there are many manipulations of independent variables that change response latency (e.g., Chase, 1984; Donders, 1869/1969; Posner, 1978; Wickens, 1984); for the purposes of the present discussion, memory search (Sternberg, 1966, 1969) serves as a prototypical paradigm for the examination of experimental latency variation.

Random latency variation affects evoked-response measures as well as reaction time, and it may have the same sources. The latency of underlying brain events has been shown to vary with reaction time (Kutas et al., 1977). Kutas et al. demonstrated that in a categorization task, P300 latency and reaction time were correlated $(r=.61)$ when subjects were instructed to maintain accuracy.

As an example of experimental latency variation, Adam and Collins (1978) and Ford, Roth, Mohs, Hopkins, and Kopell (1979) showed that in the Sternberg memory search task, P300 latency increased at a constant rate per item, slightly less than reaction time in the same experiments.

Either source of variation in the latency of brain events may at best reduce the effectiveness or at worst totally invalidate the use of signal averaging techniques, and it may have unusual effects on the results of PCA (Donchin \& Heffley, 1978; Hunt, 1984; Möcks, 1986). Random latency variation is probably the more tractable of the two sources, since its magnitude is likely to be smaller, and since plausible techniques may be developed to eliminate or minimize its effects. One method for reducing random latency variation of a particular component from one trial to another, for example, is to translate the time scale of each trial so that its waveform optimally agrees with a template (Kutas et al., 1977; Woody, 1967). The positive or negative translation serves to bring the latency-varying features of the waveform into alignment, but inevitably displaces others; in particular, it eliminates the synchrony of the stimulus onset on translated trials. Other monotonic distortions of the time scale of individual trials may prove to be effective in eliminating random variability in the latency of evoked-response events without interfering with subsequent treatment of the waveform as a whole in further analysis, such as expanding or contracting the time scale of trials to align these separate events. (Because pilot attempts to use such techniques on data acquired for other purposes have not shown consistent improvements of analysis, techniques for reducing the effects of random latency variability are not discussed further here.)

The effects of experimental latency variation can be large enough to translate parts of the evoked response over a large enough time range that the shapes of the waveform from different conditions are quite obviously different. This raises no problem for the peak latency and amplitude and latency measurements, since waveforms are averaged separately for each experimental condition and subject. However, PCA is often applied across conditions that have produced latency differences. The effect of PCA approaches to analysis when such conditions obtain was first explored in a simulation.

\section{SIMULATION ANALYSIS OF STAGES AND PRINCIPAL COMPONENTS ANALYSIS}

In order to determine how PCA might be affected by latency variation, simulated evoked-response data were generated that contained two different "events": one with constant latency but varying amplitude and another with varying latency but constant amplitude.

\section{Method}

Thirty trials were generated for each of six conditions in a $2 \times 3$ design. In each trial there were two half-sinusoid components with arbitrary amplitude of 10 units, embedded in random noise. There were two conditions (A1 and A2) for the first component: it was present in each trial in either positive (A1) or negative (A2) polarity and had a constant latency of $400 \mathrm{msec}$ after the beginning of each simulated sample. There were three conditions (B1, B2, B3) for the second component: it always had positive polarity, but latency varied among 600,680 , and $760 \mathrm{msec}$ for conditions B1, B2, and B3. The six conditions of the experiment corresponded to the factorial combination of the two $\mathrm{A}$ conditions and three $\mathrm{B}$ conditions. Think of variable $\mathbf{A}$ as being similar to a stimulus manipulation affecting a perceptual process (such as stimulus identification) in such a way that brain events do not vary appreciably in latency, and variable B as representing a manipulation (such as memory set size) that affects the latency of some brain events in a consistent manner from one condition to another. The interval $(80 \mathrm{msec})$ between different conditions was chosen to be representative of one that might be observed in memory search when set size was varied among 1,3 , or 5 items (see behavioral data, below). PCA was conducted in a manner similar to that used on behavioral data; that is, the component analysis was performed on the differences in each condition from the grand mean. 


\section{Component Patterns}

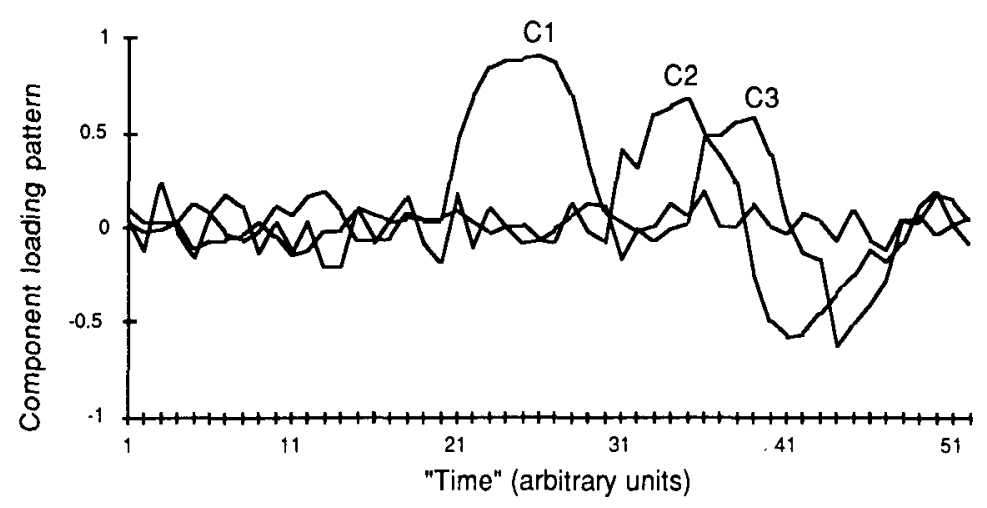

Figure 1. Components (C1, C2, and C3) extracted by principal components analysis from simulated evoked-response data.

\section{Results}

PCA of the simulated data extracted three components, and an analysis of variance (ANOVA) after varimax rotation indicated that the first component was significantly related to the first independent variable (A) and the second and third were related to the second independent variable (B) (all $p \mathrm{~s}<.001$ ). Figure 1 indicates the component loading pattern for each of the three components that were extracted by the PCA. To evaluate how well the latency-varying event is represented by the PCA solution, the data were reconstructed by linear combination, using only the second and third components, that is, the components that showed significant effects of the latencyvarying variable. Figure 2 indicates the reconstruction of three of the six experimental conditions from loading pattern and independent variable scores, for three of the memory set sizes at one intensity. The second and third components represent the latency-varying event almost perfectly. From these results one could easily infer the underlying pattern of simulated events.

\section{Discussion}

To summarize the simulation results, the event that is stationary in latency is well represented by one component (the first extracted), as expected. The latency-varying event is, however, significantly related to two components. Thus the simulation indicates that a brain event varying in latency affects more than one component of the PCA, and that, together, those components identified by ANOVA as being affected by the same independent variable(s) can be combined (using appropriate weights from the component scores calculated for each experimental condition) to reconstruct the original voltage changes produced by the independent variable manipulation(s). Conversely, when the results of PCA reveal two or more consecutive components that are affected by the same independent variable manipulations, it suggests (but does not prove) that there is a single underlying event whose latency varies in response to the relevant independent variable manipulation.

\section{STERNBERG MEMORY SEARCH}

Many of the performance situations that have been of interest to students of cognitive processes have been conceptualized in a sequential stage framework. ${ }^{1}$ Evokedresponse data from these situations carry large sources of experimental latency variation.

Previous applications (Chapman et al., 1981; Karis, Fabiani, \& Donchin, 1984) attempted to identify the components extracted from evoked-response data with particular brain events, with the goal of a one-for-one correspondence between the two. For some events this is a plausible strategy (e.g., the association of the $\mathrm{P} 300$ peak with completion of stimulus evaluation), but for other events the one-for-one correspondence between events and components will not obtain.

First, consider the underlying stages of information processing that are assumed to occur in the Sternberg memory search task (Figure 3). For simplicity, each trial is presumed (Sternberg, 1966, 1969) to consist of three separate stages of processing: stimulus identification, memory search, and response selection. For convenience, we will assume that stimulus identification is constant in duration, memory search varies in duration depending on the size of the memory set, and response selection is again constant. Now, consider what would happen if the evoked responses to all trials were compared at many latencies after the onset of the test letter in each trial, as occurs in PCA. At the time indicated by the arrow marked A, the comparison should indicate effects related to independent variable manipulations that affect the stimulus identification stage: for example, stimulus degradation or intensity manipulations. At B, such comparison would compare the early stages of memory search across conditions in which memory set size varied. If search was qualitatively different, depending on memory set size, the effect might be apparent.

At $\mathrm{C}$, comparison across conditions would be more complex. In the simplified model, at this latency after test letter onset, the comparison would cut across qualitatively 

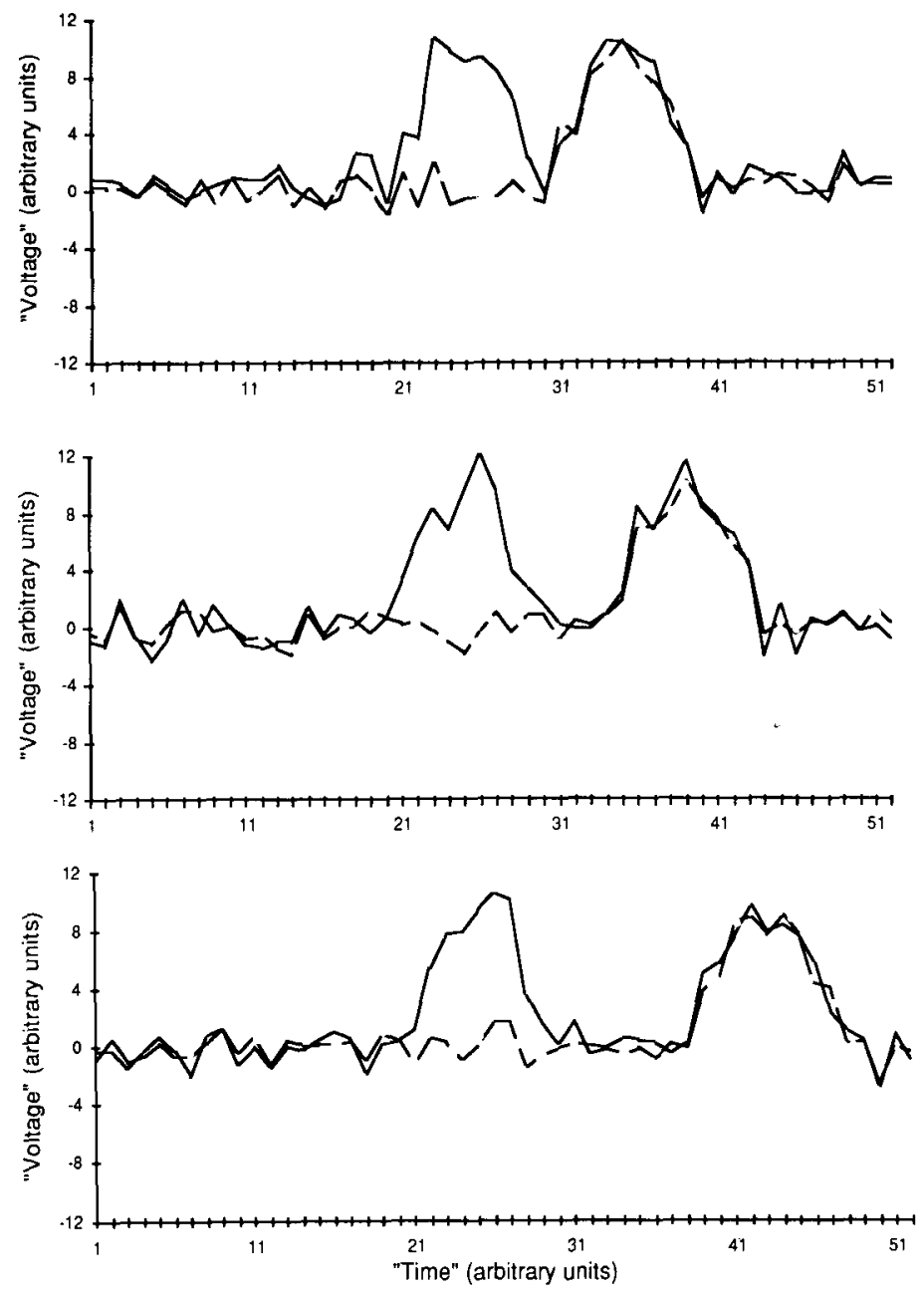

Figure 2. Reconstruction of that part of the simulated evoked response that is represented by the second and third components extracted (those that showed a significant effect of the B variable). Solid lines: simulated data for conditions A1B1 (top), A1B2 (middle), and A1B3 (bottom). Dashed lines: reconstructed data.

different processes. Evoked responses at time $\mathrm{C}$ contain events related to response selection if memory set size is 1 , those related to memory search if set size is 5 . Effects of the comparison would include those that affect memory search (such as memory set size) as well as those that affect response selection (most notably response type, yes or no). In terms of the analysis, these complex effects could appear as memory set $x$ response type interactions on components that represent this time epoch.

At $D$ in Figure 3, a latency long enough to exceed the effects of memory search, a comparison across conditions might show only the effects of response type, if these persisted in time for all conditions.

The model's use is illustrated with data from a Sternberg memory search experiment.

\section{Method}

Seven subjects were given 216 trials each of Sternberg memory search in which memory set size was varied randomly from trial to trial among the values 1,3 , and 5; and target letter intensity was high or low. On each trial, evoked responses from three scalp locations (CPZ and just anterior to P3 and P4; Jasper, 1958), referred to linked ears, were recorded for $1.2 \mathrm{msec}$ after the onset of the test letter. Evoked-response data were next averaged by condition and centered with respect to each person's grand average at each scalp location, so as to reflect only withinsubjects differences (see Möcks \& Verleger, 1985, for a similar approach to within-subjects differences, and a more fully developed rationale for it). The data were then reduced by PCA and varimax rotation and evaluated by an ANOVA of the component scores for each of the independent variables.

\section{Results}

Analysis of reaction times showed main effects of memory set $(p<.0001)$ and target intensity $(p<.05)$, but no differences due to response type (yes or no). Reaction time was $515 \mathrm{msec}$ for Memory Set Size 1, $660 \mathrm{msec}$ for Set Size 3, and $726 \mathrm{msec}$ for Set Size 5. Responses to high-intensity test items were slightly faster $(620 \mathrm{msec})$ than to low-intensity test items $(647 \mathrm{msec})$. Of $10 \mathrm{com}$ - 


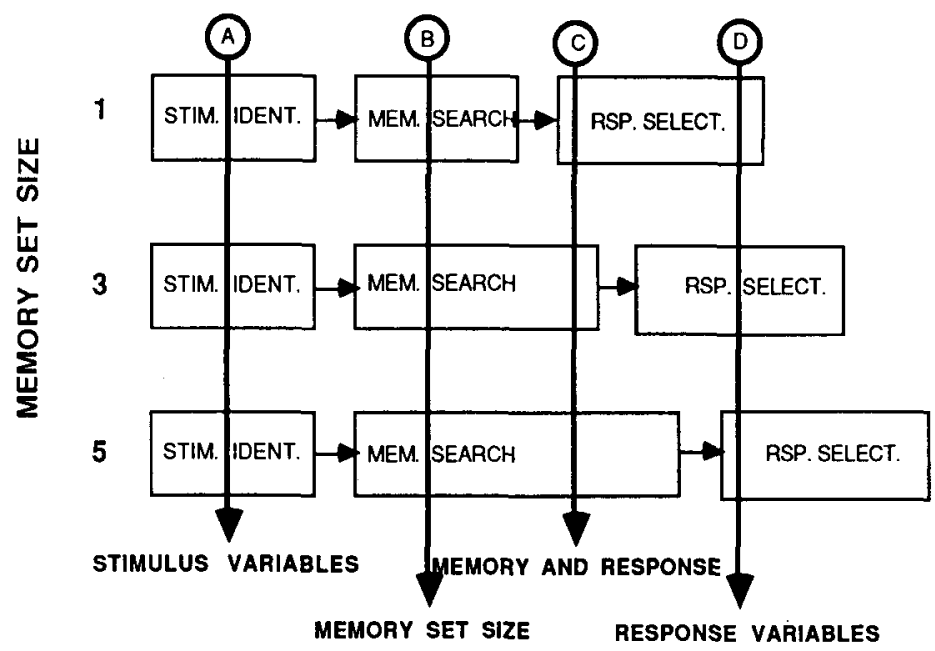

TIME FROM TEST STIMULUS ONSET

Figure 3. Hypothetical underlying stages of information processing during Sternberg memory search with memory set sizes of 1,3 , and 5 . Vertical arrows indicate times at which comparisons across conditions might show particular effects (see text).

ponents extracted by PCA (which together accounted for $38.9 \%$ of the variance in the difference waveforms), the scores of 8 showed significant effects of the independent variable manipulations. These effects are shown in $\mathrm{Ta}$ ble 1 , along with the latency after stimulus onset at which the peak of the component loading pattern occurred. (This latency is referred to below as component latency.)

The shortest latency component $\mathbf{8 0} \mathrm{msec}$ after test letter onset) was affected by a location $x$ intensity interaction. The effect of intensity is compatible with the comparison at time $\mathrm{A}$ in Figure 3. Three medium-latency components (with latencies of $320,380,460 \mathrm{msec}$ ) were affected by memory set size and response type interactions, among other complex interactions, including some with intensity. (Note that one of the components at $380 \mathrm{msec}$ was biphasic, with large pattern scores also at a latency of $740 \mathrm{msec}$. No interpretation is offered for the latter, since it occurs well after the response had occurred in nearly every condition.) These effects are consistent with the comparison at $C$ in Figure 3, as well as with other times somewhat sooner or later than $C$ that also cut across presumed search and response selection processes.

An effect on one extracted component, the location $x$ response type interaction on the component of a latency of $220 \mathrm{msec}$, does not fit well into the scheme of Figure 1. The interaction results from the larger effect of response type (yes or no) on the midline than on the lateral electrode locations. Its inconsistency with the model of Figure 1 is in the expected order of effects of the various independent variables. According to the assumed sequence of stages, effects of response type, alone or in combination with effects of memory set size, should occur only after the memory search has been completed in at least the Set Size 1 condition. However, this effect of response type occurs for a component whose latency is shorter than any that show effects of memory set, and it is not different for different set sizes.

\section{GENERAL DISCUSSION}

The simulation and behavioral data reported here indicate that principal components analysis may be a useful analytic tool in situations where independent variable manipulations cause large variations in the latency of both underlying brain events and manual response latencies.

The simulation data indicate that when such events vary in time, the manipulated variables affect more than one of the components extracted by PCA. In some cases it may be possible to infer from the pattern of effects the actual sequence and timing of underlying events.

Table 1

Results of Principal Components Analysis of Memory Search Data

\begin{tabular}{|c|c|c|c|}
\hline Component & $\begin{array}{c}\text { Peak } \\
\text { Pattern Loading } \\
\text { Latency* }\end{array}$ & $\begin{array}{c}\text { Percent } \\
\text { Variance } \\
\text { Explained }\end{array}$ & $\begin{array}{c}\text { Independent } \\
\text { Variable } \\
\text { Effects } \dagger\end{array}$ \\
\hline $\mathrm{Cl}$ & $380 ; 740$ & 7.3 & I \\
\hline $\mathrm{C} 2$ & 460 & 5.3 & $\begin{array}{c}\mathbf{M} \times \mathrm{T} \$, \mathbf{M}, \mathrm{T} \\
\mathrm{I} \times \mathbf{M} \times \mathrm{T}, \mathrm{L} \times \mathbf{I} \times \mathbf{M} \times \mathrm{T}\end{array}$ \\
\hline C3 & 920 & 5.2 & $\mathrm{~L} \times \mathrm{M} \times \mathrm{T}$ \\
\hline C5 & 320 & 4.4 & $\mathrm{M} \times \mathrm{T}$ \\
\hline C6 & $80 ; 180$ & 3.0 & $L \times I$ \\
\hline $\mathrm{C8}$ & 220 & 2.6 & $L \times T$ \\
\hline $\mathrm{C} 10$ & 380 & 1.5 & $\mathrm{~L} \times \mathrm{I} \neq, \mathrm{I} \times \mathrm{T}$ \\
\hline
\end{tabular}

*Latency of most prominent peak of loading pattern of component. Components $\mathrm{Cl}$ and $\mathrm{C} 6$ each had two prominent peaks. †Significance levels are $p<.01$ except as indicated: $\neq p<.001$ and $\S<.0001$. Key for effects: I-intensity; $M-$ memory set size; L-electrode location: $\mathrm{T}$-response type. 
In the case of the behavioral data from a Sternberg memory search task, PCA indicates not only that there is brain activity that varies with response type, but it also suggests the time epochs at which the variable affects processing. The components whose peaks lie between 320 and $460 \mathrm{msec}$ may be taken to indicate the time course of memory search and response selection, since these show effects of memory set size and response type, in various patterns of interaction. Response selection could begin at about $320 \mathrm{msec}$ at the earliest, even on trials with only a single item in the memory set. This time estimate is consistent with the observed reaction time of $480-560$ msec with only a single item in memory, because this allows $160 \mathrm{msec}$ for the response selection stage to be completed under even the conditions of the fastest manual response. This sequence (memory search followed by response selection) is perfectly consistent with reasonable expectations for this task. However, if this analysis is correct, then the early effect of response type at 220 msec must represent the effect of response type on a process different from memory search and comparison or response selection. It would complicate the interpretation of response latency data in the traditional additive factors paradigm, since it suggests that a variable (response type in this case) can affect different stages that are widely separated in time. Exactly what the early process affected by response type might be is not clear, but stimulus priming or other preattentive processes may provide plausible hypotheses (Vaughan \& Bell, 1986).

In summary, PCA of evoked-response data has contributed to the study of the sequence and timing of stages of cognitive performance. The simulation data presented here suggest how evoked responses may be analyzed when underlying stages vary in latency, and the behavioral data suggest how evoked-response analysis can elucidate both the duration of underlying stages and their time of occurrence.

\section{REFERENCES}

Adam, N., \& Collins, G. I. (1978). Late components of the visual evoked potential to search in short-term memory. Electroencephalography \& Clinical Neurophysiology, 44, 147-156.

Callaway, E. (1984). Human information-processing: Some effects of methylphenidate, age, and scopalamine. Biological Psychiatry, 19, 649-662.

Chapman, R. M., McCrary, J. W., \& Chapman, J. A. (1981). Memory processes and evoked potentials. Canadian Journal of Psychology, 35, 201-212.

Chase, W. (1984). The timing of mental acts. In E. Donchin (Ed.), Cognitive psychophysiology: Event-related potentials and the study of cognition (pp. 221-247). Hillsdale, NJ: Erlbaum.

Coles, M. G. H., Donchin, E., \& Porges, S. W. (1986). Psychophysiology. New York: Guilford Press.

Coles, M. G. H., Gratton, G., Bashore, T. R., Eriksen, C. W., \& DonCHIN, E. (1985). A psychophysiological investigation of the continuous flow model of human information processing. Joumal of $E x$ perimental Psychology: Human Perception \& Performance, 11, $529-553$.
DonchIN, E. (1984). Cognitive psychophysiology: Event-related potentials and the study of cognition. Hillsdale, NJ: Erlbaum.

Donchin, E., \& HefrLeY, E. F. (1978). Multivariate analysis of eventrelated potential data: A tutorial review. In D. Otto (Ed.), Multidisciplinary perspectives in event-related brain potential research (pp. 555-572). Washington, DC: Environmental Protection Agency. Donders, F. C. (1969). On the speed of mental processes. In W. G. Koster (Ed. and Trans.), Attention and performance II (pp. 412-431). Amsterdam: North-Holland. (Original work published 1869)

FoRD, J. M., ROTH, W. T., MOHS, R. C., HopKiNs, W. F., \& KOPELL, B. S. (1979). Event-related potentials recorded from young and old adults during a memory retrieval task. Electroencephalography \& Clinical Neurophysiology, 47, 450-459.

HunT, E. (1984). Mathematical models of the event related potential. (Tech. Rep. No. 84-1-0289). Washington, DC: Air Force Office of Scientific Research.

JASPER, H. H. (1958). The ten-twenty electrode system of the international federation. Electroencephalography \& Clinical Neurophysiology, 10, 371-375.

Karis, D., Fabiani, M., \& Donchin, E. (1984). P300 and memory: Individual differences in the von Restorff effect. Cognitive Psychology, 16, 177-216.

KUTAS, M., MCCARThY, G., \& DonCHIN, E. (1977). Augmenting mental chronometry: The $\mathrm{P} 300$ as a measure of stimulus evaluation time. Science, 197, 792-795.

MCCARThY, G., \& Donchin, E. (1981). A metric for thought: A comparison of P300 latency and reaction time. Science, 211, 77-80.

MCClelland, J. L. (1979). On the time-relations of mental processes: An examination of processes in cascade. Psychological Review, 86, 287-330.

Möcks, J. (1986). The influence of latency jitter in principal components analysis of event-related potentials. Psychophysiology, 23, 480-484.

Möcks, J., \& VERLEGER, R. (1985). Nuisance sources of variance in principal components analysis of event-related potentials. Psychophysiology, 22, 674-688.

PosNER, M. I. (1978). Chronometric explorations of mind. Hillsdale, $\mathrm{NJ}$ : Erlbaum.

Pritchard, W. S. (1981). Psychophysiology of P300. Psychological Bulletin, 89, 506-540.

Renault, B., Ragot, R., Lesevre, N., \& Remond, A. (1982). Onset and offset of brain events as indices of mental chronometry. Science, 215, 1413-1415.

STERNBERG, S. (1966). High-speed scanning in human memory. Science, $153,652-654$.

STERNBERG, S. (1969). The discovery of processing stages: Extensions of Donders' method. Acta Psychologica, 30, 276-315.

Vaughan, J., Bell, G. (1986). Preattentive target processing inferred from evoked response analysis of speeded Sternberg search. Psychophysiology, 23, 468 (abstract).

WICKENS, C. (1984). Engineering psychology and human performance. Columbus, OH: Merrill.

WOODY, C. D. (1967). Characterization of an adaptive filter for the analysis of variable latency neuroelectric signals. Medical \& Biological Engineering, 5, 539-553.

\section{NOTE}

1. For the purposes of this paper, arguments will be developed assuming a sequential stage model. Several alternatives have been proposed for this simple model (Coles, Gratton, Bashore, Eriksen, \& Donchin, 1985; McClelland, 1979), such as cascade or overlapping-stage models. However, the arguments made here are not affected by the type of underlying model assumed, as long as it is essentially sequential in nature. 